\title{
HEURISTIC AND PERFORMANCE OF SS304 AND EN8 IN COMPUTER NUMERICAL CONTROL TURNING MACHINE
}

\author{
Arjun PR \\ Research Assistant \\ SSIAR Bangalore
}

\begin{abstract}
In any machining process, apart from obtaining the accurate dimensions, achieving a good surface quality is also of utmost importance. A machining process involves many process parameters which directly or indirectly influence the surface quality of the product. Surface roughness and waviness in turning process are caused due to various parameters of which feed, speed, depth of cut are important ones. A precise knowledge of these optimum parameters would facilitate reduce the machining costs and improve product quality. Extensive study has been conducted in the past to optimize the process parameters in any machining process to have the best product. This study investigates the effects of various parameters such as depth of cut, speed and feed on the material removal rate and surface roughness of the SS304 and EN8 in a CNC turning machine. This study presents a multi-objective optimization technique, based on genetic algorithms, to optimize the cutting parameters in turning processes: cutting depth, feed and speed. Optimization of cutting parameters is one of the most important elements in any process planning of metal parts. In this project the three objective functions, cutting depth, feed and speed are simultaneously optimized to get optimum surface finish at minimum production cost and machining time.
\end{abstract}

Index Terms - CNC Turning, Stainless steel, EN8, Optimization, genetic algorithm

\section{INTRODUCTION}

A greater attention is given to surface roughness ( $\mathrm{Ra}$ ) of product by the industry these days. Surface finish has been one of the most important considerations in determining the machinability of materials. With the more precise demands of modern engineering products, the control of surface texture together with dimensional accuracy has become more important. It has been investigated that the surface texture greatly influences the functioning of the machined parts. Whatever may be the manufacturing process use, it is not possible to produce perfectly smooth surface. The imperfections and the irregularities are bound to occur. These irregularities on the surface are usually termed as surface roughness. Surface Roughness is the important factor which effect the performances of any machining operations. The factors affecting the surface roughness are the machining conditions, work piece material and the tool geometry. Some of the other factors which also affect the surface roughness, material of the work piece, type of machining, material and sharpness of cutting tool, cutting conditions ie feed, speed and depth of cut, type of coolant used. Therefore in order to obtain better surface finish the optimal machining parameters and tool geometry are to be selected. Surface roughness, an indicator of surface quality is one of the most specified customer requirements in a machining process. For efficient use of machine tools optimum cutting parameters such as speed, feed and depth of cut are required. Surface roughness are also referred from the comparisons of cutting conditions. The main determination of surface roughness as compared to cutting speed, feed, depth of cut. The selection of optimal cutting parameters, like the number of passes, depth of cut for each pass, feed and speed is a very important issue for surface roughness of every machining process.

Quality and productivity play significant role in today's manufacturing market. Quality of a product can be described by various quality attributes. The attributes may be quantitative or qualitative. In on-line quality control controller and related equipments are provided with the job under operation and continuously the quality is being monitored. In off-line quality control the method is either to check the quality of few products from a batch or lot or to evaluate the best process environment capable of producing desired quality product

Turning is the primary operation in most of the production processes in the industry. In this operation, it is an important task to select cutting parameters for achieving high 
cutting performance. The cutting parameters that determine the quality of surface, rate of metal removal and cutting performance are the cutting speed, the feed rate, and the depth of cut. These cutting conditions and the nature of the material to be cut determine the power required to take the cut. On the other hand these parameters are influential on production cost, machining time and quality of the final product. Improper selection of cutting parameters will lead to severe quality loss and increased cost of manufacturing. So these parameters needed to be optimized to obtain desired surface quality with reduced machining time and cost. So the objective is to find the optimum cutting condition to get desired surface quality..

\section{METHODOLOGY}

Among the steel grade materials Stainless steel 304 (SS 304) and EN8 have been the versatile materials for optimizing the Machining parameters regarding surface roughness. The materials used for the experimentation having some dimensional specification. Here taken the material of stainless steel rod and EN8 Steel rod for experimentation. And also having the dimensional specification with length of specimen $135 \mathrm{~mm}$ and diameter of specimen $28 \mathrm{~mm}$. The cutting parameters which relates the surface roughness ie., depth of cut, feed rate, and cutting speed affect the roughness. Both stainless steel and EN8 steel grade material is used for the experimentation.

One of the most versatile and commonly used stainless steel in the market, Grade 304 stainless steel is the most standard used alloy of this type. Essentially grade 304 is an austenitic chromium alloy which is also known as $18 / 8$ stainless as the makeup of the steel is $18 \%$ Chromium and $8 \%$ Nickel. The chromium content promotes the material considerable resistance to the effect of corrosion and oxidation. The steels need to be cold worked to generate high tensile strength. For SS 304 which are welded heavily, post weld annealing may be necessary to provide maximum corrosive resistance.

\section{Chemical composition for SS 304}

Composition ranges for 304 stainless steel

\begin{tabular}{|c|c|c|c|c|c|c|c|}
\hline Elements & $\mathrm{C}$ & $\mathrm{Mn}$ & $\mathrm{P}$ & $\mathrm{S}$ & $\mathrm{Si}$ & $\mathrm{Cr}$ & $\mathrm{Ni}$ \\
\hline $\begin{array}{c}\text { composition } \\
\%\end{array}$ & 0.08 & 2.0 & 0.045 & 0.030 & 1.0 & $18-20$ & $8-10.5$ \\
\hline
\end{tabular}

SS304 are generally used where corrosion and oxidation resistance is important. In addition they also have good creep strength. In these Chromium and Nickel are the main alloying element .Chromium forms a protective passive film and offers the necessary resistance to corrosion. The properties are dependent mainly on the chemical composition and microstructure of the material .Austenitic stainless steel grade materials is more complex in nature than others. The major elements chromium, carbon and nickel. The carbon content is as low as it commercially feasible to obtain. Chromium can range from $18 \%$ to $20 \%$ and nickel content is usually at least $8 \%$. The effect of nickel is to promote austenitic structure. The austenite structure brought about by nickel additions or in some cases nickel and manganese. Chromium has a great affinity to combine with carbon and oxygen. If the carbon gets high, the structure of stainless steel is affected and without special technique chromium in steel melting can be lost by oxidation.

Mechanical and metallurgical properties of stainless steel 304

\begin{tabular}{|c|c|c|c|c|}
\hline Property & $\begin{array}{c}\text { Tensile strength } \\
(\mathrm{MPa})\end{array}$ & $\begin{array}{c}\text { Yield strength } \\
(\mathrm{MPa})\end{array}$ & $\begin{array}{c}\text { Rockwell } \\
\text { Hardness }(\mathrm{HR})\end{array}$ & $\begin{array}{c}\text { Brinell } \\
\text { Hardness(HB) }\end{array}$ \\
\hline Value & 515 & 205 & 92 & 201 \\
\hline
\end{tabular}

Chemical composition of EN8

\begin{tabular}{|c|c|c|c|c|c|}
\hline Elements & Carbon & Silicon & Manganese & Sulphur & Phosphorus \\
\hline $\begin{array}{c}\text { Composition } \\
\%\end{array}$ & $0.40 \%$ & $0.25 \%$ & $\begin{array}{c}0.60 \text { to } \\
0.80 \%\end{array}$ & $0.050 \%$ & $0.050 \%$ \\
\hline
\end{tabular}


EN8 in its heat treated forms processes good homogenous metallurgical structures; giving consistent machining properties.EN8 is an unalloyed medium carbon steel.EN8 is a medium strength steel, good tensile strength. It is normally supplied in cold drawing or as rolled. Tensile properties can vary but are usually between $500-800 \mathrm{~N} / \mathrm{mm}^{2}$. Carbon steels are simply alloy of iron and carbon as the major strengthening agent. The carbon strengthens by solid solution strengthening, and if the carbon level is high enough, the alloy can be quench hardened. Carbon steels as steels with up to $2 \%$ carbon and only residual amounts of other elements, except those added for deoxidation with silicon limited to $0.6 \%$,copper to $0.6 \%$, and manganese to $1.65 \%$.Carbon is the main alloying element and traces of other elements like $\mathrm{Si}, \mathrm{Mn}, \mathrm{S}$ and $\mathrm{P}$ are considered as impurities. We previously defined a carbon steel as an alloy of iron, with carbon as the major strengthening element. The carbon strengthens by solid solution strengthening, and if the carbon level is enough, the alloy can be quench hardened .Carbon steels are available in all the mill forms ie bar, strip, and sheet shapes and an important selection factor pertaining to carbon steel is whether to use a cold or hot finished product. The mechanical properties of carbon steels vary significantly.

In this work as a part of experimentation the machining operation regarding turning held on CNC machine. Now a day's production of complicated components in large quantities with high accuracy requires the transfer of instructions from man to the machine through automatic devices. The control systems used with such machines require the instructions to be encoded in a suitable language, in a particular pattern and on a convenient medium, so that the machine can read these instructions automatically to perform the job. Numerical control of machine tools refers to the operation of machine tool is using numerical data. It is a technique for automatically controlling machine tools, equipment or processes. Numerical control can be defined as a form of programmable automation in which the process can be controlled by numbers, letters and symbols. These numbers, letters and symbols gathered together and logically organized to direct a machine tool for a specific task is called NC program. It is possible to use computers instead of controller units in the numerical control machine tools thereby replacing much of the conventional NC hardware with software. Such a machine having a computer assigned to one particular task to all the basic numerical control actions is known as computer numerical control machine. Thus computer numerical control system is a numerical control system in which a dedicated computer is used to perform in accordance with the control program which stored in the memory of the computer. Since the CNC machines produce parts the inspection cost can be reduced. Flexibility is the main advantage of the CNC machine. These kinds of machines need a skilled programmer to develop the programming in accordance with the parts design. In the case of CNC machine the programs enters into the memory of the controller in terms of some codes. The instructions given to the machines by these codes. Mainly two types of codes we have used in the industries. One of them which relates with the preparatory functions they may be called as $G$ codes and another relates with miscellaneous functions. The machining process under gone by the instructions by the codes which regulate the machine movement regarding the axis. Here for turning should have two axis for the control of machining movement. Actually the turning process means the cutting tools moves towards the work piece in accordance with the parameter dimension given on each axis.

Genetic algorithms are robust and adaptive methods, successfully used for solving optimization problems. They are powerful tools for the optimization of functions that can more easily locate the global optimum. The reason lies in the fact that GA seeks an optimal solution in the space of solutions, starting from groups of points, rather than a starting point. GA use only the objective function to search optimal solutions (derivatives or other additional information on the function are not necessary). The basic building block in the GA is a population of individuals, which is usually between 10 and 200. Each individual represents a possible solution of the problem. The data processed by GA are represented by an array of strings (or chromosomes) with finite length, where each bit is called allele or gene. A value of the fitness function is attached to each individual, in order to evaluate its quality. A collection of strings is called population, and the population at a certain point of time is referred as generation. The generation of the initial population of strings is done in a random way. 

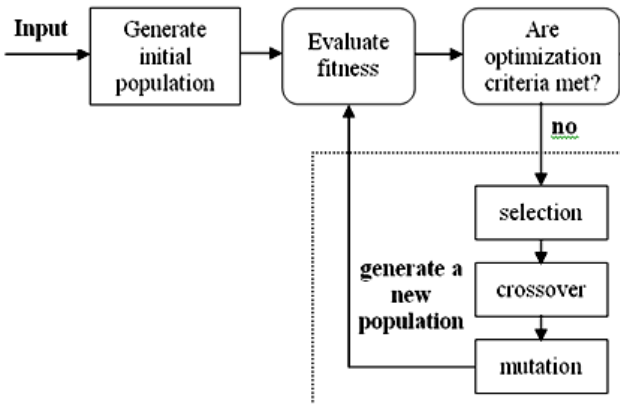

The basic operators on the genes in the chromosome are crossover and mutation. Reproduction of some selected chromosomes is a process in which certain binary strings are transformed and passed to the next generation. Selection is usually implemented through the so called process roulette wheel. The crossover is the main operator, which generates new strings, eventually with better fitness values.

After crossover, mutation is performed to ensure some randomness in the new chromosomes. In fact, even though crossover generally leads to better results, this does not bring new quality of information on the level of bits. As a source of different quality, the mutation of bits is usually performed. Mutations can lead to degenerative solutions (which probably will be soon eliminated by the process), or to a completely new solution.

\section{SPECIMENS USED FOR EXPERIMENTATION}

\section{First specimen}

Specimen material I $\quad$ Stainless steel 304

Length of the specimen $\quad \mathrm{I}-135 \mathrm{~mm}$

Diameter of the specimen I $-28 \mathrm{~mm}$

Number of pieces $\quad-9$

\section{Second specimen}

Specimen material II - EN 8 (Carbon steel)

Length of the specimen II $-135 \mathrm{~mm}$

Diameter of the specimen II $-28 \mathrm{~mm}$

Number of pieces

$$
-9
$$

The cutting parameters selected for turning operations are cutting speed (V), Feed rate (f), and Depth of cut (d). The selected range of cutting parameters and the levels at which the turning operations to be done are given in the following tables.
Levels of machining

\begin{tabular}{|c|c|c|c|}
\hline \multirow{2}{*}{ Parameters } & \multicolumn{3}{|c|}{ Levels } \\
\cline { 2 - 4 } & $\mathbf{1}$ & $\mathbf{2}$ & $\mathbf{3}$ \\
\hline Cutting Speed, N (rpm) & 700 & 1000 & 1300 \\
\hline Feed, f (mm/rev) & 0.06 & 0.08 & 0.1 \\
\hline Depth of cut, d (mm) & 0.3 & 0.5 & 0.7 \\
\hline
\end{tabular}

\section{DESIGN OF EXPERIMENTS (DOE)}

Design of Experiments (DOE) is an experimental strategy in which effects of multiple factors are studied simultaneously by running tests at various levels of the factors. Taguchi DOE is a powerful and efficient method over other traditional methods. In Taguchi DOE only 9 experiments has to be done. A series of structured tests are designed in which planned changes are made to the input variables of a process or system. The effects of these changes on a pre-defined output are then assessed. For each input variable, a number of levels are defined that represent the range for which the effect of that variable is desired to be known. The response is then measured for each run. The method of analysis is to look for differences between response (output) readings for different groups of the input changes. These differences are then attributed to the input variables acting alone (called a single effect). MiniTab 17 software was used for generating the Taguchi's orthogonal array.

\section{RESULTS AND DISCUSSIONS}

\section{SURFACE ROUGHNESS}

Single pass turning operation on both SS304 and EN8 were done and surface roughnesses of all pieces were measured using MITUTOYO surftest SJ-210. All the values obtained are given in below tables. 
Surface roughness of SS304

\begin{tabular}{|c|c|c|c|c|}
\hline \multirow{2}{*}{ Sl. } & \multicolumn{3}{|c|}{ Input Parameters } & Output parameter \\
\cline { 2 - 5 } No. & $\begin{array}{c}\text { Speed, } \mathbf{N} \\
(\mathbf{r p m})\end{array}$ & $\begin{array}{c}\text { Feed, } \mathbf{f} \\
(\mathbf{m m} / \mathbf{r e v})\end{array}$ & $\begin{array}{c}\text { Depth of cut, } \\
\mathbf{d} \\
(\mathbf{m m})\end{array}$ & $\begin{array}{c}\text { Surface Roughness, Ra } \\
(\boldsymbol{\mu m})\end{array}$ \\
\hline 1 & 700 & 0.06 & 0.3 & 0.249 \\
\hline 2 & 700 & 0.08 & 0.5 & 0.521 \\
\hline 3 & 700 & 0.10 & 0.7 & 0.476 \\
\hline 4 & 1000 & 0.06 & 0.5 & 0.247 \\
\hline 5 & 1000 & 0.08 & 0.7 & 0.176 \\
\hline 6 & 1000 & 0.10 & 0.3 & 0.151 \\
\hline 7 & 1300 & 0.06 & 0.7 & 0.221 \\
\hline 8 & 1300 & 0.08 & 0.3 & 0.171 \\
\hline 9 & 1300 & 0.10 & 0.5 & 0.243 \\
\hline
\end{tabular}

Surface roughness and MRR for EN8

\begin{tabular}{|c|c|c|c|c|}
\hline \multirow{2}{*}{$\begin{array}{r}\text { Sl. } \\
\text { No. }\end{array}$} & \multicolumn{3}{|c|}{ Input Parameters } & Output Parameter \\
\cline { 2 - 5 } & $\begin{array}{c}\text { Speed, N } \\
(\mathbf{r p m})\end{array}$ & $\begin{array}{c}\text { Feed, } \mathbf{f} \\
(\mathbf{m m} / \mathbf{r e v})\end{array}$ & $\begin{array}{c}\text { Depth of cut, } \\
\mathbf{d} \\
\mathbf{( m m})\end{array}$ & $\begin{array}{c}\text { Surface Roughness, Ra } \\
(\boldsymbol{\mu m})\end{array}$ \\
\hline 1 & 700 & 0.06 & 0.3 & 1.866 \\
\hline 2 & 700 & 0.08 & 0.5 & 1.598 \\
\hline 3 & 700 & 0.10 & 0.7 & 1.485 \\
\hline 4 & 1000 & 0.06 & 0.5 & 1.313 \\
\hline 5 & 1000 & 0.08 & 0.7 & 1.333 \\
\hline 6 & 1000 & 0.10 & 0.3 & 1.611 \\
\hline 7 & 1300 & 0.06 & 0.7 & 2.269 \\
\hline 8 & 1300 & 0.08 & 0.3 & 2.483 \\
\hline 9 & 1300 & 0.10 & 0.5 & 2.037 \\
\hline
\end{tabular}

Genetic algorithm conditions and parameter constraints for surface roughness of $\mathrm{SS304}$

In Matlab tool box, Genetic algorithm conditions and parameter constraints for surface roughness is shown below.

Population size $=50$

Number of variables $=3$

Initial range $=[700,0.06,0.3 ; 1300,0.1,0.7]$

Selection function $=$ Roulette

Elite count $=2$

Crossover fraction $=0.8$

Mutation function = Uniform
Mutation rate $=0.2$

Migration $=$ forward

Total number of iterations $=10000$

Level of Display = Iterative

Predicted output from genetic algorithm for surface roughnessofEN8
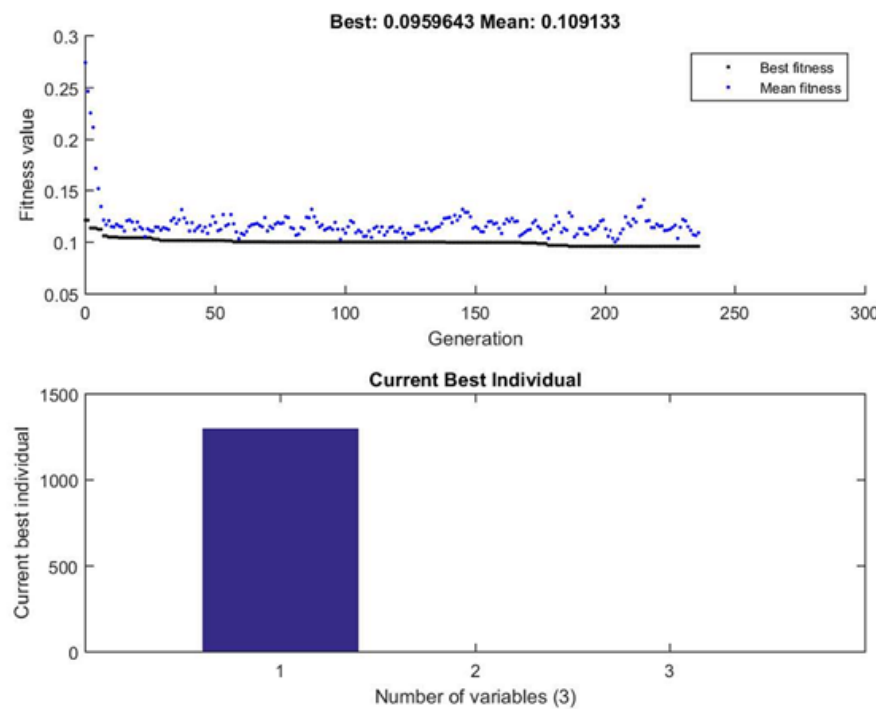

Genetic algorithm conditions and parameter constraints for surface roughness of $\mathrm{SS304}$

In Matlab tool box, Genetic algorithm conditions and parameter constraints for surface roughness is shown below.

Population size $=50$

Number of variables $=3$

Initial range $=[700,0.06,0.3 ; 1300,0.1,0.7]$

Selection function $=$ Roulette

Elite count $=2$

Crossover fraction $=0.8$

Mutation function $=$ Uniform

Mutation rate $=0.2$

Migration $=$ forward

Total number of iterations $=10000$

Level of Display = Iterative

Predicted output from genetic algorithm for surface roughnessof EN8 
The following are the predicted output from genetic Average value of surface roughness for SS304, Ra $=0.0957 \mu \mathrm{m}$ algorithm using Matlab software for surface roughness
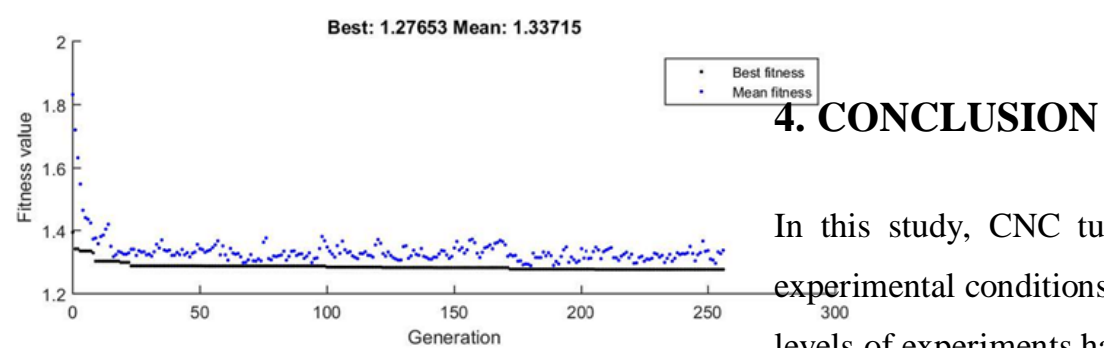

In this study, CNC turning operation is done under various experimental conditions and surface roughness was measured. 9 levels of experiments had been done. The most effecting factors

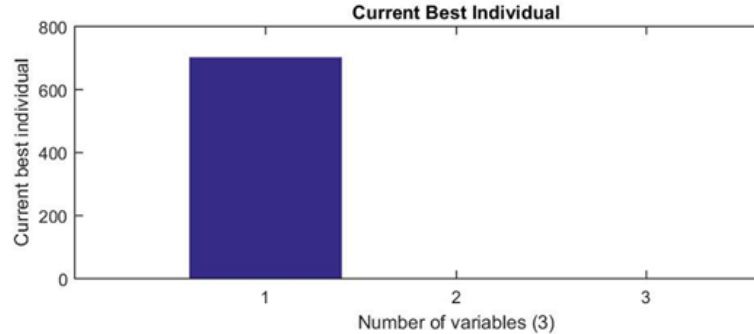
on surface roughness are speed and depth of cut. From the regression equation the output parameters can be optimized for any machine with different combination of input parameters.

In the present work, the cutting conditions such as speed, feed

Validation results for $\mathrm{SS304}$

\begin{tabular}{|c|c|c|c|c|}
\hline \multirow{2}{*}{ Sl. No. } & \multicolumn{3}{|c|}{ Input Parameter } & Output parameter \\
\cline { 2 - 5 } & $\begin{array}{c}\text { Speed, N } \\
\mathbf{r p m}\end{array}$ & $\begin{array}{c}\text { Feed, f } \\
\mathbf{m m} / \mathbf{r e v}\end{array}$ & $\begin{array}{c}\text { Depth of cut, d } \\
\mathbf{m m}\end{array}$ & $\begin{array}{c}\text { Surface Roughness, Ra } \\
\boldsymbol{\mu m}\end{array}$ \\
\hline 1 & 1299.446 & 0.06 & 0.302 & 0.0957 \\
\hline 2 & 1299.446 & 0.06 & 0.302 & 0.0958 \\
\hline 3 & 1299.446 & 0.06 & 0.302 & 0.0957 \\
\hline
\end{tabular}

Average value of surface roughness for SS304, $\mathrm{Ra}=0.0957 \mu \mathrm{m}$

\section{Validation results for EN8}

\begin{tabular}{|c|c|c|c|c|}
\hline \multirow{2}{*}{ Sl. No. } & \multicolumn{3}{|c|}{ Input Parameter } & Output parameter \\
\cline { 2 - 5 } & $\begin{array}{c}\text { Speed, N } \\
\mathbf{~ r p m}\end{array}$ & $\begin{array}{c}\text { Feed, f } \\
\mathbf{m m} / \mathbf{r e v}\end{array}$ & $\begin{array}{c}\text { Depth of cut, d } \\
\mathbf{m m}\end{array}$ & $\begin{array}{c}\text { Surface Roughness, Ra } \\
\boldsymbol{\mu m}\end{array}$ \\
\hline 1 & 702.377 & 0.1 & 0.699 & 1.275 \\
\hline 2 & 702.377 & 0.1 & 0.699 & 1.276 \\
\hline 3 & 702.377 & 0.1 & 0.699 & 1.276 \\
\hline
\end{tabular}
and depth of cut are optimized based on surface finish as objective function. Whereas, there are other constraints also such as Material Removal Rate, dimensional accuracy, rigidity and reliability of the system etc. that could also be considered. Here the optimization is done only for the single-pass turning operation that can also be further extended to the multi-pass operations. Tool selection, machine selection, process selection and tool path selection are the other important areas for optimization in process planning. Besides this, the sequencing and scheduling optimization are the other important areas for the GA implementation. Also the other advanced GA operators for reproduction, crossover and mutation could be applied to the optimization problem.

\section{REFERENCE}

[1] Basil M. Eldhose, Cijo Mathew, Dr. Binumarkose, "Optimization of the Cutting Parameters of SS 304 for CNC Turning Operation", International Journal of Innovative Research in Advanced Engineering (IJIRAE) ISSN: 2349-2163 Volume 1 Issue 8 (September 2014) www.ijirae.com 
[2] Durairaja M, S. Gowri, "Parametric Optimization for Improved Tool Life and Surface Finish in Micro Turning using Genetic Algorithm”, International Conference on Design And Manufacturing, IConDM 2013.

[3] Ganesan H, G.Mohankumar, K.Ganesan, K.Ramesh Kumar, "Optimization of machining parameters in turning process using genetic algorithm and particle swarm optimization with experimental verification", H.Ganesan et al. / International Journal of Engineering Science and Technology (IJEST)

[4] MahendraKorat, NeerajAgarwal, "Optimization of Different Machining Parameters of En24 Alloy Steel In CNC Turning by Use of Taguchi Method", MahendraKorat, NeerajAgarwal / International Journal of Engineering Research and Applications (IJERA) ISSN: 2248-9622 www.ijera.com Vol. 2, Issue 5, September- October 2012, pp.160-16

[5] Rahul Dhabalea, VijayKumar S. Jattib, T.P.Singh, "Multi-Objective Optimization of Turning Process during Machining of $\mathrm{AlMg} 1 \mathrm{SiCu}$ Using NonDominated Sorted Genetic Algorithm", 3rd International Conference on Materials Processing and Characterisation (ICMPC 2014)

[6] Routara B C, A K Sahoo, A K Parida, P C Padhi, "Response surface methodology and Genetic Algorithm used to determine the optimum cutting conditions leading to minimum surface roughness in CNC turning operation on EN-8 steel", International conference on modeling, optimization and computing, (ICMOC, 2012)

[7] Saravanakumar K, M.R.Pratheesh Kumar, Dr.A.K.ShaikDawood, “Optimization of CNC Turning Process Parameters on INCONEL 718 Using Genetic Algorithm", IRACST - Engineering Science and Technology: An International Journal (ESTIJ), ISSN: 2250-3498, Vol.2, No. 4, August 2012.

[8] Senthil Kumar A, M. Adam Khan, and R. Thiraviam, "Machining Parameters Optimization For Alumina Based Ceramic Cutting Tools Using Genetic Algorithm", Machining Science and Technology, 10:471-489 Copyright \# 2006 Taylor \& Francis Group, LLC ISSN: 1091-0344 print/1532-2483 online DOI: 10.1080/10910340601009358.

[9] Subramanian M, M.Sakthivel, K.Sooryaprakash, R.Sudhakaran, "Optimization of Cutting Parameters for Cutting Force in Shoulder Milling of Al7075-T6
Using Response Surface Methodology and Genetic Algorithm", International Conference on Design And Manufacturing, IConDM 2013.

[10] Thangarasu V. S., G. Devaraj, R. Sivasubramanian, "High speed CNC machining of AISI 304 stainless steel; Optimization of process parameters by MOGA", International Journal of Engineering, Science and TechnologyVol. 4, No. 3, 2012, pp. 66-77 